\title{
Zwischen Neo- und Postfunktionalismus: Die Integrationstheorien und die Eurokrise'
}

\author{
Frank Schimmelfennig
}

\section{Between Neo- and Postfunctionalism: Integration Theories and the Euro Crisis}

Abstract: The euro crisis poses a fundamental challenge to the European Union but also to the theories of European integration. Whereas intergovernmentalism provides the best explanation of the original design of Economic and Monetary Union, it is losing explanatory power in the crisis. The intergovernmental logic of integration based on exogenous preferences and state bargaining power gives way to the neofunctionalist logic. Transnational interdependence, autonomous supranational organizations, endogenous preferences, path dependency, and spillover drive the process and have produced more integration in fiscal policy. As expected by postfunctionalism, however, the crisis politicizes European integration and increases Euro-skeptic mobilization at the same time, thus putting into danger the further integration and cohesion of the Eurozone. So far, however, the neofunctionalist logic has prevailed.

Keywords: Economic and Monetary Union, Euro Crisis, European Integration, Integration Theory

Schlagwörter: Eurokrise, Europäische Integration, Integrationstheorie, Wirtschafts- und Währungsunion

\section{Einleitung}

Als das irische Volk den Vertrag von Lissabon im Oktober 2009 in einem zweiten Referendum guthiess, hatte es den Anschein, als ob die EU nach einem Jahrzehnt der stürmischen Erweiterung und einer an Rückschlägen reichen Kette von Vertragsrevisionen endlich zur Ruhe kommen würde. Im gleichen Monat begann jedoch die Staatschuldenkrise Griechenlands, die sich zu einer Krise der Eurozone entwickelte und die europäische Politik seit nunmehr fast drei Jahren - und ohne absehbares Ende - in Atem hält.

Bisher kannte die europäische Integration nur eine Richtung: vorwärts. Zwar gab es Phasen relativer Stagnation (wie in den 1970er-Jahren), Blockaden und Verzögerungen wie bei den gescheiterten Volksabstimmungen zum Verfassungsvertrag, oder differenzierte Integration wie bei den „Opt-outs“ Dänemarks und Großbritanniens aus der Währungsunion. Nie wurde jedoch eine integrierte Politik renationalisiert; nie verließ ein Land einen integrierten Politikbereich. In der Schuldenkrise drohte nun ausgerechnet die Währungsunion auseinanderzubre-

1 Ich danke der PVS-Redaktion für die Einladung zu diesem Beitrag und Christoph Elhardt, Sandra Lavenex, Thomas Schäubli und Thomas Winzen für hilfreiche Kommentare. 
chen, das wichtigste Integrationsprojekt und der am stärksten integrierte Politikbereich der EU. Damit ist die Eurokrise die wahrscheinlich größte Bewährungsprobe in der Geschichte der europäischen Integration.

Auch für die Theorien der europäischen Integration bedeutet die Eurokrise einen harten Test. Gegenstand dieser Theorien ist die Verschiebung politischer Kompetenzen von der nationalen auf die supranationale Ebene. Sie erklären die Bedingungen und Mechanismen der Integration, ihre Antriebs- und Bremskräfte und ihre Dynamik. Nicht die Krise selbst und ihre Ursachen stehen im Mittelpunkt integrationstheoretischer Erklärungen, sondern die politischen Reaktionen und institutionellen Konsequenzen. Wer sind die massgeblichen Akteure und welche Interessen verfolgen sie? Wie und mit welchem Ergebnis verhandeln sie miteinander? Vor allem aber: führt die Krise zu mehr oder weniger Integration, und woran liegt das? In der Krise sollte besonders gut sichtbar werden, was die EU zusammenhält und vorantreibt, aber auch, wo die Hindernisse und Grenzen supranationaler Integration in Europa liegen.

Lange Zeit ist es um die Theorien der europäischen Integration eher still gewesen. Zum einen galt die seit den 1960er-Jahren bestehende Konkurrenz von Neofunktionalismus und Intergouvernementalismus als ausgereizt und abgestanden. Zum anderen schien die Entwicklung der EU an einem stabilen konstitutionellen Gleichgewichtspunkt angelangt zu sein (Moravcsik 2005); das politikwissenschaftliche Interesse an der EU verschob sich vom Integrationsprozess zur „multilevel governance“, der Politikentwicklung innerhalb eines relativ stabilen Mehrebenensystems (Jachtenfuchs u. Kohler-Koch 1996; Marks et al. 1996). Theorien „normaler“ Politik aus der vergleichenden und Policy-Analyse lösten die Integrationstheorien als Leittheorien der Europaforschung ab (Hix 1994). Die Krise zeigt jedoch, dass die EU noch immer ein instabiles und unfertiges politisches Gebilde ist. Mit einem Mal kommt das Gefüge der Kompetenzverteilung in Europa wieder in Bewegung.

Aktuell ist ein Test der Integrationstheorien allerdings mit einem äußerst beweglichen Ziel konfrontiert. Bei Abgabefrist dieses Artikel spitzte sich die Eurokrise mehr denn je zu: Austrittsszenarien für Griechenland und Bankenkrise in Spanien einerseits; Pläne für eine Banken- und Fiskalunion andererseits. Angesichts des offenen Ergebnisses muss der Theorievergleich daher vor allem die Entwicklung der Jahre 2010-2012 und ihre Zwischenergebnisse ins Auge nehmen.

Meine zentrale These lautet: Der Intergouvernementalismus, der die anfängliche Ausgestaltung der Wirtschafts- und Währungsunion (WWU) noch schlüssig erklären konnte, verliert in der Krise an Erklärungskraft. Die auf exogenen Präferenzen und staatlicher Verhandlungsmacht beruhende intergouvernementalistische Integrationslogik wird abgelöst durch die neofunktionalistische Logik: endogene Regierungspräferenzen, transnationale Verflechtung, supranationale Autonomie, Pfadabhängigkeit und Spillover treiben die Entwicklung. Das ursprüngliche Design der WWU wurde von den Regierungen der großen Mitgliedstaaten ausgehandelt; Deutschland setzte sich aufgrund seiner Verhandlungsmacht dabei weitgehend durch; einer zentralisierten Geldpolitik mit einer regierungsunabhängigen Europäischen Zentralbank (EZB) stand eine dezentrali- 
sierte Fiskalpolitik ohne Beistands- und Ausgleichsmechanismen und mit lediglich intergouvernementaler Kontrolle der Staatsverschuldung gegenüber.

In der Eurokrise treiben die „Finanzmärkte“ die Regierungen vor sich her. Angesichts der „sunk costs“ der Währungsunion und der hohen Risiken ihres Zerfalls wurde die Verteidigung des Euro über alle nationalen Differenzen bei der Spar- und Beistandspolitik hinweg zur obersten Präferenz aller Euroländer. Das schränkt auch die deutsche Verhandlungsmacht ein. Widerwillig hat die Bundesregierung hinnehmen müssen, dass das Beistandsverbot durch die Rettungsschirme und Interventionen der EZB aufgeweicht wurde. Die einseitige Integration der Geldpolitik ließ sich nicht halten; die Zentralisierung der Fiskalpolitik schreitet voran. Damit stützt die Eurokrise die supranationalistische Integrationstheorie.

Allerdings ist offen, ob sich die neofunktionalistische Integrationslogik am Ende durchsetzen kann oder aber von der durch Politisierung und Identitätspolitik bestimmten postfunktionalen Logik ausgebremst wird. Zum einen schränkt die Schwäche der europäischen Identität die transnationale Haftungsbereitschaft und Solidarität ein. Zum anderen formiert sich Widerstand von unten gegen die technokratisch verordnete Sparpolitik: die ursprünglich als unpolitisch konstruierte Währungsunion wird politisiert; die Euroskepsis wächst.

Im weiteren Verlauf des Artikels gebe ich zunächst einen Überblick über die Integrationstheorien des Intergouvernementalismus, Supranationalismus und Konstruktivismus. Es folgt eine Rekapitulation der Entstehung der Währungsunion, bevor ich mich den Präferenzen und Konflikten sowie der Verhandlungsdynamik und den Verhandlungsergebnissen in der Eurokrise zuwende. Einige integrationstheoretische Schlussfolgerungen beschließen den Beitrag.

\section{Die Integrationstheorien}

Für die Darstellung der Integrationstheorien beschränke ich mich auf die Kernannahmen und -hypothesen von drei Theoriefamilien: Intergouvernementalismus, Supranationalismus und Konstruktivismus (Leuffen et al. 2012). Dabei handelt es sich um die maßgeblichen Teilnehmer an der Theoriedebatte, mit denen sich die wesentlichen Integrationsentwicklungen in der Eurokrise erfassen lassen. Auf die Binnendifferenzierung dieser Theoriefamilien kann ich ebenso wenig eingehen wie auf andere Ansätze (vgl. z. B. Bieling u. Lerch 2006).

\subsection{Intergouvernementalismus}

Der Intergouvernementalismus (Moravcsik 1993, 1998) sieht in den Regierungen der Mitgliedstaaten die zentralen Akteure des Integrationsprozesses. Sie vertreten das nationale Interesse: im realistischen Intergouvernementalismus die Autonomie und Sicherheit des Staates, im liberalen Intergouvernementalismus die - in erster Linie ökonomischen - Präferenzen der durchsetzungsfähigsten nationalen Interessengruppen. In der Geldpolitik geht es allerdings vorrangig um die makroökonomischen Präferenzen der Regierungen (Moravcsik 1998, S. 3).

Auf der Basis ihrer nationalen Interessen verhandeln die Regierungen über die Integration. Damit es zu einer Vereinbarung über den Transfer nationaler Kompe- 
tenzen kommen kann, müssen die Regierungen ein Interesse an Integration haben. Das ist zum einen der Fall, wenn sie sich von der Integration einen Zuwachs an Autonomie und/oder Wohlstand erwarten - von einer gemeinsamen Währung also z. B. eine höhere Widerstandskraft gegenüber Währungsspekulationen oder den Abbau von Wechselkursrisiken. Zum anderen bedarf es übereinstimmender Vorstellungen über die Ausgestaltung der Integration: die politikfeldspezifischen Regeln und die Kompetenzen supranationaler Organisationen.

Stimmen diese Vorstellungen nicht überein, setzen sich die Regierungen mit der größten Verhandlungsmacht durch. Die Verhandlungsmacht ist eine Funktion asymmetrischer Interdependenz: Je weniger ein Staat die anderen braucht und je mehr diese ihn brauchen, um die Integrationsziele zu erreichen, desto eher kann er durchsetzen, dass die Integration zu seinen Bedingungen erfolgt. Auch innenpolitischer Widerstand stärkt die Verhandlungsmacht: Wer es zu Hause mit starken Gegnern der Integration zu tun hat, kann die anderen Regierungen zu Konzessionen bewegen, um die Ratifikation der Vereinbarungen sicherzustellen (Moravcsik 1993, S. 514-517).

Der Intergouvernementalismus geht von einer funktionalen Institutionentheorie aus, der zufolge internationale Institutionen von Staaten geschaffen werden, um Probleme bei der internationalen Kooperation durch die autoritative Auslegung der Regeln und die Aufdeckung und Sanktionierung von Regelverletzungen zu verringern (Keohane 1984). Sie handeln also im Auftrag und Interesse der Staaten. Überhaupt nimmt der Intergouvernementalismus an, dass der Integrationsprozess nicht nur im Interesse der Staaten verläuft, sondern auch unter ihrer Kontrolle bleibt. Staatliche Präferenzen bleiben exogen, werden also durch den Integrationsprozess nicht verändert.

\subsection{Supranationalismus}

Der Supranationalismus steht in direkter Nachfolge des Neofunktionalismus, der Theorie europäischer Integration aus der Anfangszeit der Europäischen Gemeinschaften (Haas 1968; Stone Sweet u. Sandholtz 1997). Was den Start der Integration angeht, unterscheiden sich Supranationalismus und Intergouvernementalismus nicht grundlegend. Paul Pierson etwa stimmt zu, dass der erste Integrationsschritt das Ergebnis von intergouvernementalen Verhandlungen ist und die relative Verhandlungsmacht der beteiligten Staaten reflektiert (1998, S. 29). Der Supranationalismus bestreitet aber, dass die Regierungen den weiteren Verlauf der Integration antizipieren und kontrollieren. Der Supranationalismus folgt dabei dem historischen Institutionalismus (vgl. z. B. Pierson u. Skocpol 2002): Institutionen mögen zum Zeitpunkt ihrer Entstehung effizient sein und die Interessen ihrer Gründer widerspiegeln. Anschließend entwickeln sie jedoch eine Eigendynamik, die sie von der anfänglichen Interessenkonstellation entfernt, und eine Beharrungskraft, die sie gegen veränderte Interessen und Effizienzanforderungen immunisiert.

Zwei Mechanismen spielen dabei eine zentrale Rolle: Spillover (Haas 1968; Schmitter 1969) und Pfadabhängigkeit (Pierson 1998). Funktionaler Spillover ist eine Konsequenz des inkrementellen, sektoralen europäischen Integrationsstils, 
der dazu führt, dass einzelne Politikbereiche supranational reguliert werden, während andere in nationaler Kompetenz verbleiben. Aufgrund der Interdependenzen zwischen den Politikbereichen entstehen dabei negative Externalitäten: Die supranationale Regulierung bleibt wegen der nationalen Regulierung verknüpfter Politikbereiche suboptimal, und die nationale Politik büßt Effizienz ein. Institutioneller Spillover ist eine Konsequenz der Delegation von Kompetenzen an supranationale Organe. Diese Organe nutzen die ihnen zugewiesenen Befugnisse auch dazu, die Integration eigenständig voranzutreiben und die eigenen Kompetenzen zu erweitern.

Die Regierungen stehen diesen Entwicklungen nicht ohnmächtig gegenüber. Sie haben grundsätzlich die Möglichkeit, die Kompetenzen supranationaler Organe zurückzustutzen oder integrierte Politikbereiche zu renationalisieren. Pfadabhängigkeit erklärt, warum sie diese Möglichkeit nicht nutzen (können). Zum einen sind die Regeln der Vertragsänderung restriktiv: sie erfordern Konsens unter den Regierungen und nationale Ratifikation in jedem Mitgliedsland. Wenn nur eine Minderheit der Staaten mit dem Status quo zufrieden ist, lässt er sich nicht ändern. Zum anderen haben Regierungen und private Akteure hohe Kosten auf sich genommen, um sich der Integration anzupassen. Angesichts dieser „sunk costs“ schwindet ihre Bereitschaft, aus der Integration auszusteigen oder neue Pfade einzuschlagen. Der Status quo wird Bestandteil der nunmehr endogenen Regierungspräferenzen. Außerdem schafft die Integration neue oder stärkt bestehende Interessengruppen, die sich gegen eine Renationalisierung wehren. Unter diesen Umständen sind die Regierungen gezwungen, institutionellen Spillover hinzunehmen, und entscheiden sich beim funktionalen Spillover für die Integration weiterer Politikbereiche.

\subsection{Konstruktivismus}

Der Konstruktivismus ist die jüngste und am wenigsten kanonisierte unter den drei Integrationstheorien (vgl. Schimmelfennig 2012). Er hebt die ideellen Voraussetzungen und Bedingungen der Integration hervor. Schlüsselkonzept ist die Identität. Der Konstruktivismus teilt die Annahme des soziologischen Institutionalismus, dass Organisationen Gemeinschaften repräsentieren, die sich auf gemeinsame Policy-Ideen, Werte, Normen und kollektive Identitäten stützen. Integration setzt einerseits gemeinsame oder zumindest kompatible Ideen und Identitäten voraus; andererseits stärkt sie die internationale Gemeinschaft, indem sie gemeinsame Ideen und Identitäten institutionalisiert und Regierungen und Gesellschaften sozialisiert.

Je konsensfähiger die Gemeinschaftsideen sind, je belastbarer die kollektive Identität ist, und je mehr Institutionalisierung und Sozialisation zur Stärkung der ideellen Ressourcen der Integration beitragen, desto mehr Integration ist möglich. Die Währungsunion beruht demnach sowohl auf gemeinsamen Policy-Ideen über die „richtige“ Geld- und Fiskalpolitik als auch auf Einstellungen der beteiligten Gesellschaften, die es erlauben, einen Kernbereich nationaler Souveränität und Identität zu integrieren. 
Gemeinschaftliche Ideen, Werte und Normen ermöglichen zudem Integrationsergebnisse, die rein auf der Basis der intergouvernementalen Interessen- und Machtkonstellation nicht zu erklären sind. Die „Konsensnorm“ erleichtert die Entscheidungsfindung in einem auf breite Mehrheiten angewiesenen Entscheidungsverfahren (Lewis 2010) und die „Demokratienorm“ prägt sowohl die Erweiterung der EU als auch ihre Parlamentarisierung (Schimmelfennig 2010). Andererseits können ideelle Faktoren Integrationsergebnisse verhindern, die aus Sicht der Regierungen funktional sind. Das gilt vor allem, wenn die Integration sich auf Politikbereiche erstreckt, die für die Identität der nationalen Gesellschaften konstitutiv sind, oder wenn die Zentralisierung eines Politikbereichs transnationales Vertrauen und transnationale Solidarität übermäßig strapaziert.

In ihrer „postfunktionalen Theorie der europäischen Integration“ behaupten Hooghe und Marks, dass der intergouvernementalistische und supranationalistische Fokus auf Eliten, Interessengruppen und wirtschaftliche Interessen in der Anfangsphase der europäischen Integration gerechtfertigt war. Seit den 1990erJahren erstreckt sich die Integration jedoch zunehmend auf identitätsrelevante Politikbereiche und greift tief in die Lebensumstände ein. Damit geht eine Politisierung der europäischen Integration einher; der „ermöglichende Konsens“ der Anfangsjahre wandelte sich zum „beschränkenden Dissens“. Europäische Integration wird zu einer Identitätsfrage und bietet populistischen Parteien die Chance zu Wahlerfolgen zu kommen. Länder, in denen exklusiv nationale Identitäten stark verbreitet sind und institutionelle Verfahren (wie z. B. Referenden über EUVerträge) die europaskeptische Mobilisierung begünstigen, sind davon besonders betroffen (Hooghe u. Marks 2008). Während die neofunktionalistische Logik in Spillover-Situationen das Einfallstor für eine anfänglich nicht gewünschte Vertiefung der europäischen Integration sieht, vermutet der Postfunktionalismus identitätsbedingte Grenzen und Rückschläge einer elitären, technokratischen und effizienzorientierten Zentralisierung.

\section{Das Design der WWU: intergouvernementaler Konflikt und deutsche Verhandlungsmacht}

Die Wirtschafts- und Währungsunion, die im Vertrag von Maastricht 1991 vereinbart wurde, zeichnet sich durch eine Kombination von zentralisierter Geldpolitik und dezentralisierter Fiskalpolitik aus. Die Geldpolitik ist vollständig supranational integriert. Die Euroländer haben sämtliche geldpolitischen Kompetenzen an das Eurosystem abgetreten, an dessen Spitze die EZB steht. Die EZB ist sowohl von den Mitgliedstaaten als auch von den Organen der EU unabhängig (Art. 130 AEUV $^{2}$ ); sie besitzt das alleinige Recht, die Geldpolitik der Eurozone festzulegen und durchzuführen; und sie ist vertraglich verpflichtet, dabei vorrangig die Preisstabilität zu gewährleisten (Art. 127 AEUV).

Der Vertrag von Maastricht begründete jedoch keine Fiskalunion. Die EU kann keine eigenen Steuern und Abgaben erheben und bezieht ihre Haushaltsmittel im

2 Vertrag über die Arbeitsweise der Europäischen Union. 
Wesentlichen aus Zuwendungen der Mitgliedstaaten. Sie darf sich auch nicht verschulden. Der EU-Haushalt liegt bei lediglich etwa einem Prozent der Wirtschaftsleistung der EU - die Staatshaushalte der Mitgliedstaaten hingegen bei 40-50\% (und mehr). Damit ist der fiskalische Handlungsspielraum der EU extrem eingeschränkt. Ein Finanzausgleich existiert nicht; es werden lediglich strukturschwache Regionen über die Regionalpolitik der EU gefördert. Entsprechend der Nichtbeistandsklausel (Art. 125 AEUV) besteht ein Haftungsausschluss der Union und der anderen Mitglieder für Verbindlichkeiten eines Mitgliedstaats. Nach Art. 123 ist es der EZB verboten, Staaten zu finanzieren oder deren Schuldtitel zu erwerben.

Allerdings reguliert die EU die Haushaltspolitik der Mitgliedstaaten. Grundlage dafür ist das Verfahren zur Vermeidung übermäßiger öffentlicher Defizite (Art. 126 AEUV). Für die Euroländer wurde 1997 zusätzlich der Stabilitäts- und Wachstumspakt vereinbart. Er schreibt die Verschuldungsgrenzen aus den Beitrittskriterien der Währungsunion für deren Mitglieder fort: eine jährliche Neuverschuldung von maximal drei Prozent des Bruttoinlandsprodukts (BIP) und eine Gesamtverschuldung von maximal 60 Prozent des BIP. Wird die Neuverschuldungsgrenze überschritten, leitet die Europäische Kommission ein mehrstufiges Verfahren ein, an dessen Ende die Finanzminister der Euro-Gruppe mit qualifizierter Mehrheit und ohne Beteiligung des betroffenen Landes finanzielle Sanktionen verhängen können. Damit handelt es sich im Kern um ein intergouvernementales Verfahren. Mit der Reform des Stabilitäts- und Wachstumspakts von 2005 wurde es durch zusätzliche Ausnahmesituationen und längere Fristen für Defizitsünder noch entschärft; finanzielle Sanktionen wurden nie verhängt.

Der Intergouvernementalismus bietet die beste Erklärung für die anfängliche Ausgestaltung der WWU (Moravcsik 1998, Kap. 6). Die Währungspolitik in Europa war traditionell durch den Gegensatz von Hart- und Weichwährungsländern geprägt - repräsentiert durch Deutschland mit einer von der unabhängigen Bundesbank durchgesetzten Politik der Geldwertstabilität und durch Frankreich, dessen Regierung in wirtschaftlichen Krisenzeiten mit der Abwertung des Franc und der Ausweitung der Geldmenge die Konjunktur zu beleben versuchte. Im Verlauf der 1980er-Jahre erschwerte die zunehmende Kapitalmobilität jedoch die makroökonomische Politik der Weichwährungsländer; im Binnenmarktprogramm von 1986 verabredeten die Mitgliedstaaten zudem die Abschaffung der Kapitalverkehrskontrollen in der Gemeinschaft. Frankreich verpflichtete sich einem „starken Franc“, und die Inflationsrate lag am Ende des Jahrzehnts nur geringfügig über der deutschen. Diese makroökonomische Konvergenz machte eine Währungsunion prinzipiell möglich. Konflikte über die Ausgestaltung der WWU bestanden jedoch fort und konnten erst in intergouvernementalen Verhandlungen vor allem zwischen Deutschland und Frankreich - entschieden werden (Dyson u. Featherstone 1999, S. 14).

Frankreich (unterstützt von Belgien und Italien) wollte eine intergouvernementale WWU, in der die Regierungen die Geldpolitik kontrollieren würden und die keine wirtschaftliche Konvergenz voraussetzte. Deutschland (unterstützt von den Niederlanden) vertrat hingegen das Prinzip einer unabhängigen, der Inflationsbekämpfung verpflichteten Zentralbank und machte realwirtschaftliche und geldpolitische Konvergenz zu einer Voraussetzung der Währungsintegration. 
Entscheidend für das Ergebnis war die unterschiedliche Verhandlungsmacht Deutschlands und Frankreichs. Zum einen hatte Frankreich ein weitaus größeres Interesse an der Währungsunion als Deutschland. Das EWS hatte sich in den 1980er-Jahren zu einer D-Mark-Zone entwickelt, deren Mitglieder sich den antiinflationären geldpolitischen Präferenzen und Vorgaben der Bundesbank anpassen mussten, ohne auf diese Politik Einfluss nehmen zu können. Die Währungsintegration bot Frankreich daher die Möglichkeit, Autonomie zurückzugewinnen. ${ }^{3}$ Frankreich hoffte, in einer Währungsunion seine Kapitalmarktkosten senken zu können; Deutschland fürchtete das Gegenteil.

Zum anderen war der Handlungsspielraum der deutschen Regierung eingeschränkt. Die Bundesbank hatte bei einer Veränderung des Status quo am meisten zu verlieren; entsprechend skeptisch waren ihre Vertreter gegenüber den europäischen Plänen. Gleichzeitig genoss sie großes Vertrauen in der Gesellschaft. Eine Währungsunion gegen den Widerstand der Bundesbank und in Abkehr von den Prinzipien der Unabhängigkeit und der Geldwertstabilität wäre daher innenpolitisch nur schwer durchzusetzen gewesen. Die führende Rolle des Finanzministeriums und die Beteiligung der Bundesbank an den Verhandlungen halfen, diese Bedenken zu zerstreuen, und verschafften neoliberalen Prinzipien und makroökonomischer Expertise ein Gegengewicht zu den integrationspolitischen Zielen von Bundeskanzler Helmut Kohl und des Außenministeriums (Dyson u. Featherstone 1999, S. 372-374, 449).

Die größere Zufriedenheit mit dem Status quo und der engere innenpolitische Spielraum verliehen der deutschen Regierung eine überlegene Verhandlungsmacht (Wolf u. Zangl 1996). Wenn die französische Regierung die Währungsunion wollte, dann musste sie die deutschen Bedingungen dafür akzeptieren: die Konvergenzkriterien, die Unabhängigkeit der Zentralbank und das Primat der Geldwertstabilität. Genauso wichtig war es für Deutschland, diese Bedingungen auch für die Zukunft zu sichern. Dafür bedurfte es eines institutionellen Designs, das die politischen und finanziellen Begehrlichkeiten der Weichwährungsländer verlässlich abwehren würde.

Zum einen wurden dafür die Unabhängigkeit der EZB und das Primat der Preisstabilität vertraglich verankert; sie können also gegen Deutschlands Willen nicht verändert werden. Zum anderen bestand Deutschland mit Erfolg auf der Nichtbeistandsklausel und der Regulierung der Staatsverschuldung im Stabilitätsund Wachstumspakt, um dem „moral hazard“-Problem zu entgehen, das aus einer Währungsunion mit dezentralisierter Fiskalpolitik erwächst. Es war zu befürchten, dass die wirtschaftlich schwächeren Euroländer die günstigeren Kreditbedingungen der Währungsunion für eine expansive Schuldenpolitik nutzen würden, anstatt den politisch schwerer durchsetzbaren Weg der Anpassung durch höhere Steuern, geringere Staatsausgaben oder niedrigere Löhne zu gehen. Am Ende könnten sie darauf spekulieren, vor dem Staatsbankrott durch direkte Transfers der anderen Euroländer oder durch eine inflationstreibende Niedrig-

3 Vgl. Dyson u. Featherstone 1999, S. 97; Grieco 1996. Parsons (2003, Kap. 7) zeigt, dass diese Position Mitterrands in Frankreich durchaus umstritten war. 
zinspolitik der EZB bewahrt zu werden. Dieses Problem wurde in der Eurokrise virulent.

\section{Integration in der Eurokrise: Pfadabhängigkeit und Spillover in die Fiskalpolitik}

Dass der Intergouvernementalismus das Design der Währungsunion überzeugend erklärt, muss nicht heißen, dass er auch die Folgewirkungen angemessen erfasst. Ohnehin nehmen Supranationalismus und Konstruktivismus an, dass Spillovers und Pfadabhängigkeiten sowie Politisierung und Euroskepsis erst infolge der anfänglichen Integration greifen. Um die relative Erklärungskraft der Integrationstheorien in der Eurokrise zu prüfen, analysiere ich zunächst die Präferenzen der Integrationsakteure und dann den Verlauf und die Zwischenergebnisse der Verhandlungen.

\subsection{Präferenzen: sparen, retten, den Euro verteidigen}

Auf den ersten Blick spiegeln die Regierungspräferenzen in der Eurokrise die Präferenzkonstellation der Entstehungsphase der Währungsunion und die fiskalische Position der Euroländer wider. Die zentralen Präferenzdimensionen sind fiskalischer Beistand und fiskalische Kontrolle. Der zentrale Konflikt verläuft zwischen denjenigen, die möglichst viel Kontrolle und Sparsamkeit verlangen und dabei möglichst wenig Beistand leisten wollen (Sparen), und denjenigen, die Beistand fordern und sich dabei möglichst wenig einschränken wollen (Retten).

Wenig überraschend handelt es sich bei den „Sparern“ um die relativ gering verschuldeten Staaten, die hohe Bonität genießen und deshalb für ihre Staatsschulden nur geringe Zinsen aufwenden müssen. Sie müssen daher keine schmerzhaften Sparanstrengungen unternehmen und brauchen keinen externen Beistand. Andererseits sträuben sie sich gegen Haftungs- und Beistandsverpflichtungen gegenüber den hoch verschuldeten Euroländern und verlangen von ihnen strikt kontrollierte und sanktionierte Sparmaßnahmen. Mit Deutschland an der Spitze haben sich Finnland, die Niederlande, Österreich und Schweden regelmäßig gegen Euro-Bonds und die Ausweitung der Rettungsschirme und für automatische Sanktionen gegen Defizitsünder ausgesprochen. Auch die neuen Euroländer, die mit hoher fiskalischer Disziplin den Beitritt zur Eurozone erreicht haben, gehören dazu. Damit hat sich die deutsch-niederländische Koalition aus der Entstehungsphase der Währungsunion um Länder mit ähnlicher makroökonomischer Orientierung erweitert. Das Ideal dieser Koalition lässt sich auch mit dem Stichwort „Stabilitätsunion“ kennzeichnen.

Fürs „Retten“ sind die höher verschuldeten Euroländer, die unter der Zinsbelastung und den Sparauflagen leiden. Sie fordern daher großzügige Kreditgarantien durch die Euroländer bis hin zu einer Vergemeinschaftung von Staatsschulden und eine lockere Sparpolitik. Zur dieser Koalition gehören Belgien, Frankreicht, Griechenland, Italien, Portugal und Spanien. Sie hat sich gegen harte, automatische Sanktionen für Defizitsünder und für die Aufstockung der Rettungsschirme, unbegrenzte Anleihekäufe durch die EZB sowie eine Banklizenz für die EFSF ausgesprochen (mit ähnlicher Wirkung wie Anleihekäufe). Unter Präsi- 
dent Sarkozy war Frankreich zunächst gegen Euro-Bonds, Präsident Hollande befürwortet sie hingegen und fordert wachstumsfördernde Maßnahmen begleitend zu den Sparauflagen. Diese Gruppe steht für den Einstieg in die „Transferunion“, die von Deutschland vehement abgelehnt wird. Sie entspricht der von Frankreich angeführten Koalition aus der Entstehungszeit der Währungsunion. Wie damals fordert sie eine „Wirtschaftsregierung“ für die Eurozone, die auch die Zentralbank anleitet.

Soweit deckt sich die Empirie mit der intergouvernementalistischen Erwartung exogener und auf strukturellen ökonomischen Positionen beruhenden Regierungspräferenzen. Nachzutragen ist noch, dass die Europäische Kommission erwartungsgemäß die föderalistischste Position einnimmt: sie wünscht eine weitreichende supranationale Kontrolle der nationalen Haushalte und eine (teilweise) Vergemeinschaftung von Staatsschulden durch Euro-Bonds. Claude Juncker, Premier Luxemburgs und Vorsitzender der Eurogruppe, liegt auf der gleichen Linie.

Das ist aber nicht das gesamte Bild. Hinzu kommt ein überragendes Interesse aller beteiligten Akteure am Fortbestand des Euro - und der Eurozone in ihren jetzigen Grenzen. Kein Euroland stellt die Währungsunion in Frage. Auf der einen Seite haben die Regierungen der Schuldenländer stets betont, in der Eurozone bleiben zu wollen. Auf der anderen Seite hat Bundeskanzlerin Merkel immer wieder erklärt, die Währungsunion sei das unverzichtbare Kernstück der europäischen Integration: „Europa scheitert, wenn der Euro scheitert.“4 Man werde "alles tun“, um den Euro zu verteidigen. ${ }^{5}$ Auch nach der griechischen Parlamentswahl vom Mai 2012 bekräftigte sie unisono mit den anderen Regierungen der Euroländer den Wunsch, dass Griechenland in der Eurozone bleiben möge. ${ }^{6}$

Dieses gemeinsame Interesse der Euroländer (und der EU-Mitglieder insgesamt) ist eine endogene Präferenz, wie sie der Supranationalismus als typisch für die Dynamik der europäischen Integration ansieht, also eine Präferenz, die sich erst im Anschluss an einen Integrationsschritt bilde und diesen Integrationsschritt in die Staatsräson übernimmt. Einerseits spricht die Politik offen die „Konstruktionsfehler“ an, „die bei der Schaffung der Wirtschafts- und Währungsunion begangen wurden ". ${ }^{7}$ Auch scheint es Gemeingut zu sein, dass Griechenland der Währungsunion nicht hätte beitreten dürfen. Kontrafaktisch kann man annehmen, dass die Regierungen der 1990er-Jahre mit dem Wissen des Jahres 2010 weder die Maastrichter Konstruktion der WWU noch den Beitritt Griechenlands beschlossen hätten. Andererseits zogen die Regierungen des Jahres 2010 daraus nicht die Konsequenz, den Euro abzuschaffen oder Griechenland zum Austritt zu drängen. Die Ursache für diese endogene Präferenz liegt in der Pfadabhängigkeit der Währungsintegration.

Die Währungsunion enthält zum einen beträchtliche „sunk costs“ für Staaten, Unternehmen und Bürgerinnen, die mit der Umstellung auf eine neue Währung

4 Regierungserklärung vom 27.2.2012 (http://www.tagesschau.de/wirtschaft/bundestaggriechenland108.html).

5 http://www.angela-merkel.de/page/103_686.htm.

6 Süddeutsche Zeitung, 15.5.2012, S. 1.

7 Angela Merkel, Regierungserklärung vom 14.12.2011, vgl. http://www.bundesregierung.de/Content/DE/Regierungserklaerung/2011/2011-12-14-merkel-ergebnisse-eu-rat.html. 
und eine supranationale Geldpolitik verbunden sind. Zum anderen verstärkte die Währungsunion die transnationale Kapitalverflechtung und damit die Interdependenz unter den Euroländern. Ein Auseinanderbrechen der Währungsunion - und schon das Ausscheiden eines relativ kleinen Mitgliedslandes wie Griechenland wäre mit extrem hohen Kosten und Risiken verbunden. Die Kosten und Risiken der ökonomischen Interdependenz haben die Gründer der Währungsunion außerdem durch institutionelle Hürden noch einmal gesteigert. Es gibt kein geordnetes Verfahren des Ausschlusses eines Landes aus der Eurozone, und ein Austritt kann vertragskonform nur über den Austritt aus der EU erfolgen.

Für ein Euroland wie Griechenland würde das Ausscheiden aus der Eurozone mit Sicherheit den Staatsbankrott bedeuten, mit hoher Wahrscheinlichkeit den Zusammenbruch des Geld- und Finanzsystems, Hyperinflation und das Ende externen Kapitalzuflusses, und eventuell den Kollaps des Wirtschaftslebens und den Verlust der EU-Mitgliedschaft - all dies mit unabsehbaren Folgen für Staat und Gesellschaft. Ein Staatsbankrott in einem Euroland würde als Folge der transnationalen Interdependenz auch den Finanzsektor in den stabileren Euroländern in Bedrängnis bringen. Vor allem aber fürchten die Euro-Regierungen Ansteckungseffekte: Schiede ein Land aus, wäre das Vertrauen der Anleger in die Eurozone insgesamt erschüttert; andere Euroländer kämen unter zusätzlichen Druck; ein massiver (und möglicherweise die Fähigkeiten der Eurozone übersteigender) Kapitaleinsatz wäre erforderlich. Auch für die besser gestellten Euroländer wie Deutschland wäre ein Zusammenbruch der Eurozone mit einer Aufwertung der Währung, einem Einbruch der Exporte und in der Folge einer erheblichen und lang andauernden Rezession verbunden - von den internationalen politischen Konsequenzen ganz zu schweigen. ${ }^{8}$ Angesichts dieser Kosten und Risiken räumen die Regierungen der Euroländer dem Fortbestand der Eurozone oberste Priorität ein. Das überragende Interesse aller Regierungen an der Verteidigung der Eurozone überlagert die Differenzen zwischen Sparern und Rettern.

Die endogene, durch Pfadabhängigkeit bedingte Präferenz für die Verteidigung des Euro und ihre Priorität gegenüber den exogenen, von der nationalen fiskalischen Situation bedingten Interessen der Mitgliedsländer widersprechen den Annahmen des Intergouvernementalismus. Sie sind jedoch auch nicht mit konstruktivistischen Sozialisationserwartungen vereinbar. Das gemeinsame Interesse der Euroländer beruht nicht auf konvergierenden makroökonomischen Policy-Ideen; die „Solidarität“, von der Bundeskanzlerin Merkel regelmäßig spricht, entspringt nicht einer positiven kollektiven Identität, sondern einer durch die gemeinsame Währung geschmiedeten Not- und Zweckgemeinschaft. Die Präferenzentwicklung in der WWU deckt sich daher am besten mit supranationalistischen Erwartungen.

8 Vgl. die Szenarien z. B. in Straubhaar (2011, S. 30-31) und The Economist, 26.5.2012, S. 26-27. 


\subsection{Der Verhandlungsprozess: ein Feiglingsspiel}

Die Präferenzkonstellation in der Eurokrise entspricht der eines „Feiglingsspiels“, in dem zwei Autofahrer aufeinander zurasen und verliert, wer als erster ausweicht. ${ }^{9}$ Beide haben ein überragendes Interesse daran, den Crash zu vermeiden. Einem Unentschieden (beide bremsen ab oder weichen aus) ziehen sie es allerdings vor, dass der andere nachgibt. Die „Sparer“ wollen, dass die Defizitsünder ihre Schulden selbst unter Kontrolle bringen; die „Retter“ setzen auf externen Beistand.

Die Verhandlungsdynamik des Feiglingsspiels ist so, dass beide Seiten vermeiden „auszuweichen“, so lange es geht, und sich gegenseitig Signale der Entschlossenheit senden. Da beide Seiten voneinander wissen, dass ein rationaler Gegenspieler am Ende den Crash scheuen wird, ist es strategisch sinnvoll, den Gegner von der eigenen Irrationalität oder Handlungsunfähigkeit zu überzeugen - also, um bei der Metapher des Feiglingsspiels zu bleiben, das Lenkrad aus dem Fenster zu werfen. Allerdings ist die Eurokrise kein ganz symmetrisches Feiglingsspiel: die Sparer haben bessere Chancen als die Schuldenländer, den Crash zu überstehen, fahren also gewissermaßen mit Airbag. Andererseits können die Schuldenländer glaubwürdig mit ihrer Handlungsunfähigkeit gegenüber den Finanzmärkten drohen. Deutschland ist zwar für die Rettung der Schuldenländer unverzichtbar - das verschafft der Bundesregierung die Macht, auf Sparanstrengungen, Reformen und Sanktionen zu bestehen. Gerade weil Deutschland unverzichtbar für die Rettung des Euro ist, steht die Bundesregierung aber in herausragender Verantwortung „alles zu tun“: Deutschland kann sich nicht verstecken. Das schwächt die deutsche Verhandlungsmacht. Außerdem wächst mit jeder Rettungsaktion die Haftungssumme der Gläubigerstaaten - und damit ihre Bereitschaft, weiter zu retten, um keine Verluste realisieren zu müssen.

Für diese Verhandlungsdynamik lässt sich in der Eurokrise reiche Evidenz finden. Einerseits konnte Deutschland aufgrund seiner Verhandlungsmacht zusammen mit der Sparer-Koalition und der Kommission eine Verschärfung und Zentralisierung der Budgetkontrolle durchsetzen. Der sogenannte „Sixpack“ von Rechtsakten vom Dezember 2011 sieht unter anderem vor, dass nicht nur die jährliche Neuverschuldung unter 3 Prozent bleiben, sondern auch ein Gesamtschuldenstand von über 60 Prozent des BIP verringert werden muss. Die Mitgliedsländer müssen nun ihre Haushaltsplanung der Europäischen Kommission vorlegen und Planungsvorgaben einhalten, die ein übermäßiges Defizit verhindern sollen. Im Fall des Verstoßes gegen die Schuldengrenzen treten Sanktionen schneller und automatischer in Kraft als bisher. Bußgelder müssen schon zu Beginn des Verfahrens als Pfand hinterlegt werden. Neu gilt auch die „umgekehrte Mehrheitsentscheidung “: während vorher ein qualifizierter Mehrheitsbeschluss des Rats notwendig war, um finanzielle Sanktionen in Kraft zu setzen, bedarf es nun einer qualifizierten Mehrheit, um sie zu stoppen.

Außerdem konnte Deutschland den Fiskalpakt durchsetzen. Er verpflichtet die Staaten zu einem nahezu ausgeglichenen Haushalt, ansonsten tritt automatisch

9 Zur Eurokrise als Feiglingsspiel vgl. auch Belke (2011) oder Enderlein (2012). 
eine nationale „Schuldenbremse“ in Aktion. Dieser Mechanismus muss in der Verfassung oder einem Gesetz gleichen Rangs verankert werden. Kommen Staaten dieser Verpflichtung nicht innerhalb eines Jahres nach, entscheidet der Europäische Gerichtshof bindend. Widersetzt sich ein Staat dieser Entscheidung, werden Geldstrafen verhängt. Wer den Fiskalpakt nicht ratifiziert, ist von Darlehen des Europäischen Stabilitätsmechanismus (ESM) ausgeschlossen. Neue Kompetenzen für die Kommission, bindende Entscheidungen des EuGH und die umgekehrte Mehrheitsregel machen aus einem im Kern intergouvernementalen ein weitgehend supranationales Verfahren der Kontrolle nationaler Schuldenpolitik.

Andererseits hat sich die Bundesregierung, wenn es um den Beistand für die Schuldenländer ging, immer wieder lange geziert - um dann schliesslich doch nachzugeben. Mitte März 2010 droht Bundeskanzlerin Angela Merkel noch mit der „ultima ratio“, Länder aus dem Euro-Raum auszuschliessen und zögert Finanzhilfen für Griechenland hinaus. Eine Woche später gilt die neue „ultima ratio“, Kredite zu gewähren, um den Euro zu stabilisieren, falls Länder sich am Markt nicht mehr refinanzieren können. ${ }^{10}$ Als die griechische Regierung im April 2010 einen Antrag auf Finanzhilfe stellt, bremst Deutschland. Im Mai 2010 vereinbaren IWF, EU und EZB mit Griechenland einen Kredit von 110 Milliarden Euro. Kurz darauf schaffen die Euroländer die Europäische Finanzstabilisierungsfazilität (EFSF), eine private Gesellschaft, die Anleihen begibt, deren Erlöse sie den Schuldenländern unter Sparauflagen zur Verfügung stellt. Die hochsolventen Euroländer haften für die EFSF.

Anfänglich liegt die Obergrenze für EFSF-Kredite bei 440 Milliarden Euro. Im Januar 2011 lehnt Deutschland mit der Sparer-Koalition eine von Belgien geforderte Aufstockung des Rettungsschirms ab. Im März kommt die Aufstockung. Seit Juli 2011 kann die EFSF auch Staatsanleihen von privat erwerben - nachdem Deutschland zunächst dagegen war. Im August 2011 lehnt die Bundesregierung die von Kommissionspräsident Barroso vorgeschlagene erneute Aufstockung des EFSF ab - im September stimmt der Deutsche Bundestag auf Antrag der Bundesregierung einer Erhöhung der Kreditsumme auf 780 Milliarden Euro zu.

Die EFSF soll ab 2012 durch den Europäischen Stabilitätsmechanismus (ESM) mit einer Darlehenskapazität von 500 Milliarden Euro abgelöst werden. Anders als die EFSF ist der ESM eine permanente internationale Finanzinstitution. Er besitzt eigenes Grundkapital in Höhe von 80 Milliarden Euro, das von den Mitgliedstaaten eingezahlt und bei Bedarf erhöht wird; er kann Staatsanleihen der Mitgliedsländer direkt erwerben und Kredite zur Rekapitalisierung von Banken vergeben. Beschlüsse können mit qualifizierter Mehrheit gefasst werden. Im Februar 2012 muss ein Sondergipfel abgesagt werden, weil Deutschland die Aufstockung des ESM ablehnt; im März stimmt die Bundesregierung zu, dass EFSF und ESM zunächst parallel laufen, was die Darlehenssumme auf 800 Milliarden Euro erhöht. Im Juli räumt Merkel die deutsche Position, dass die finanzielle Unterstützung von Banken über Darlehen an die verantwortlichen Staaten zu erfolgen

$10 \mathrm{Vgl}$. http://www.euractiv.de/finanzen-und-wachstum/artikel/merkels-ultima-ratio-rauswurf-auseuro-zone-002866; http://www.euractiv.de/europa-2020-und-reformen/artikel/merkels-neues-ultima-ratio-milliarden-fr-griechenland-002895. 
habe; nun soll eine direkte Unterstützung von Banken durch den ESM ermöglicht werden (die die Staatsverschuldung nicht erhöht).

Die wichtigste Linie, die (noch) hält, ist Deutschlands Widerstand gegen jede Form der Vergemeinschaftung von Schulden, sei es durch Euro-Bonds, sei es durch einen europäischen Schuldentilgungsfonds. Außerdem wies Bundeskanzlerin Merkel angesichts der Spanien-Krise im Juni 2012 darauf hin, dass „auch Deutschlands Kräfte [...] nicht unbegrenzt" seien - ein dem Feiglingsspiel angemessenes Signal beschränkter Handlungsfähigkeit, als die EU-Partner die deutsche Regierung zu stärkerem Engagement drängten. ${ }^{11}$

Die Schuldenländer haben ihrerseits versucht, die von Deutschland geforderten Kontrollen ihrer Haushaltspolitik und harten Sparauflagen zu umgehen. Irland, Portugal und Spanien mussten regelrecht unter den Rettungsschirm gedrängt werden; Zypern versuchte mit russischen Krediten der EFSF zu entgehen; Spanien, die internationale Kontrolle auf den Bankensektor zu begrenzen. Die Strategie der griechischen Syriza-Partei, den Euro zu behalten, aber das „Memorandum“ der Sparziele aufzukündigen, war ebenfalls durchaus eine rationale Strategie im Feiglingsspiel - unter der Voraussetzung, dass der Rest der Eurozone einen „Grexit“ für zu risikoreich hält. Im Vorfeld der Wahl baute die Sparer-Koalition deshalb eine eindrucksvolle Drohkulisse auf, um die griechischen Wähler und Parteien davon zu überzeugen, dass ein Austritt Griechenlands für die Eurozone, nicht aber für Griechenland, verkraftbar sei - und zwangsläufig erfolge, wenn die Sparauflagen nicht erfüllt würden.

Welche Rolle spielen andere Akteure als die Regierungen in diesem Prozess? Transnationale Akteure sind ohne Frage die treibenden Kräfte der Integrationsentwicklung in der Krise. Ohne das sich in hohen Zinssätzen ausdrückende Misstrauen der Anleger und der Rating-Agenturen wären weder die Schuldenländer bereit, sich den Sparauflagen zu unterwerfen, noch die Geberländer, Kredite zur Abwendung von Staatsbankrotten zu vergeben. Das „Vertrauen der Märkte“ wiederzugewinnen und die Abhängigkeit von ihnen zu reduzieren, ist das Ziel der Krisenpolitik.

Unter den supranationalen Akteuren spielen weder Parlament noch Gerichtshof eine relevante Rolle. Die Kommission hat zwar in der Krise ständig neue Initiativen lanciert (und die Alleingänge Deutschlands und Frankreichs kritisiert); Erfolg hatten diese aber nur, wenn sie mit der deutschen Position übereinstimmten. Während die Vorschläge der Kommission zur Verschärfung der Schuldenkontrolle inzwischen Gesetz sind, hat Deutschland die wiederholten KommissionsInitiativen für gemeinsame Anleihen der Euroländer immer aufs Neue abgelehnt. Das widerspricht aber nicht supranationalistischen Annahmen, weil diese Akteure in der WWU weder über starke Kompetenzen noch über wichtige Ressourcen verfügen. Kommission und Gerichtshof erhalten erst durch den "Sixpack“ und den Fiskalpakt bedeutende Kompetenzen.

Der relevante supranationale Akteur in der Eurokrise ist die EZB, und sie nutzte die Kompetenzen und Unabhängigkeit, die Deutschland ihr verschafft hat, zu

11 http://www.faz.net/aktuell/politik/regierungserklaerung-der-kanzlerin-auch-deutschlands-kraeftesind-nicht-unbegrenzt-11785295.html. 
einer Politik, die den ebenfalls von Deutschland durchgesetzten vertraglichen Bestimmungen widersprach. Zum einen erwarb die EZB seit Mai 2010 im Rahmen eines „Wertpapierkaufprogramms“ Staatsanleihen der hochverschuldeten Euroländer im Wert von weit über 200 Milliarden Euro und senkte damit deren Refinanzierungskosten. Im Dezember 2010 verdoppelte die EZB ihr Grundkapital nahezu, nicht zuletzt, um das Ausfallrisiko von Staatsanleihen abzudecken. Zum anderen stellte die EZB im Dezember 2011 und Februar 2012 den Geschäftsbanken im Rahmen ihrer „langfristigen Refinanzierungsoperationen“ (LRTOs) mehr als 1 Billion Euro für drei Jahre zu einem äußerst günstigen Zinssatz von 1 Prozent zur Verfügung. Mit dieser Politik des „billigen Geldes“ pumpte die EZB Liquidität in den gefährdeten Bankensektor, schuf aber gleichzeitig einen Anreiz für die Banken, hochverzinste Staatsanleihen zu kaufen. In der Folge sanken die Renditen für Staatsanleihen der hochverschuldeten Euroländer.

Offiziell wurden diese Massnahmen nicht als - vertragswidrige - Finanzierung von Staatsschulden deklariert, sondern als Förderung des reibungslosen Funktionierens der Zahlungssysteme, die zu den vertraglichen Verpflichtungen der EZB gehört. Außerdem erwarb die EZB die Anleihen nicht direkt von den Regierungen, sondern auf dem Sekundärmarkt. Erleichtert wurde die Abkehr von der „reinen Lehre" dadurch, dass der Zentralbankrat mit Mehrheit entscheidet, die deutschen Vertreter also überstimmt werden konnten. Hier zeigt sich aus deutscher Sicht eine Lücke im institutionellen Design, die die autonome Entwicklung der EZB erleichtert (Moravcsik 1998, S. 445). Mit dem designierten EZB-Vorsitzenden Weber und EZB-Chefvolkswirt Stark verzichteten zwei führende deutsche Vertreter der orthodoxen Linie auf ihre Ämter in der Zentralbank. In der zugespitzten Krise des Sommers 2011, als Italien und Spanien akut gefährdet schienen, stimmte Deutschland den Anleihekäufen durch die EZB allerdings stillschweigend $\mathrm{zu}$.

Bisher mündete das wiederholte Feiglingsspiel in eine Serie von Kompromissen; der Crash konnte vermieden werden. Die Schuldenländer haben strikte Sparauflagen und eine Verschärfung und Zentralisierung der Haushaltskontrolle akzeptiert; Deutschland und die Sparer-Koalition haben ihnen dafür mit Hunderten von Milliarden Euro beigestanden. Und die EZB ist mit Anleihekäufen und billiger Liquidität eingesprungen, wenn die Bereitschaft und die Möglichkeiten der Regierungen nicht ausreichten. Die gemeinsame endogene Präferenz der EuroRettung hat sich also durchgesetzt. Dabei ist Deutschland typischerweise immer im letzten Moment und nur soweit „ausgewichen“, wie es nötig war. Dennoch haftet Deutschland mit EFSF und ESM im schlimmsten Fall etwa in Höhe des jährlichen Bundeshaushalts, während es noch offen ist, ob und wann die verschärften Kontrollmaßnahmen des Sixpacks und des Fiskalpakts greifen werden. Weder die eingeschränkte Verhandlungsmacht Deutschlands noch das autonome und von den vertraglichen Aufgaben abweichende Verhalten der EZB stimmen mit intergouvernementalistischen Erwartungen überein.

Die bisherigen Entwicklungen in der Eurokrise entsprechen damit weitgehend der supranationalistischen Theorie. Die Reform der WWU folgt einem SpilloverProzess. Die aus der Diskrepanz von supranationaler Geldpolitik und intergouvernementaler Fiskalpolitik resultierenden negativen Externalitäten wurden in 
Richtung einer (allerdings immer noch vergleichsweise moderaten) Zentralisierung der Fiskalpolitik aufgelöst, weil eine Renationalisierung der Geldpolitik durch Pfadabhängigkeiten verstellt ist. Die zentralen handelnden Akteure sind zwar Regierungen, doch agieren sie auf der Basis endogener Präferenzen, unter dem Druck transnationaler Akteure des Finanzmarkts und flankiert durch die EZB.

\subsection{Grenzen der Pfadabhängigkeit? Die postfunktionale Herausforderung}

Die Krisenbewältigung der Euroländer war vornehmlich technokratisch ausgerichtet. Die Reform des Stabilitäts- und Währungspakts stärkt die Kommission; die Schuldenbremse des Fiskalpakts schränkt den Handlungsspielraum demokratisch gewählter Institutionen ein. Die Krisenpolitik der EZB unterliegt keiner demokratischen Legitimation und Kontrolle; der ESM ist ein reines Exekutivorgan.

Drei Ereignisse im Oktober und November 2011 illustrieren die Unterordnung der Politik unter den Sachzwang ebenfalls. Zuerst opferte die slowakische Regierungschefin Radicova ihr Amt der EFSF. Nur die Zustimmung der Slowakei fehlte noch, damit die EFSF-Erweiterung in Kraft treten konnte. Nachdem eine erste Abstimmung im slowakischen Parlament wegen Differenzen unter den Regierungsparteien gescheitert war, versprach die Ministerpräsidentin der Opposition Neuwahlen, um deren Stimmen für die Ratifikation zu gewinnen. Dabei war es sehr wahrscheinlich, dass Radicova die Wahlen verlieren würde - was später auch geschah. Die Slowakei ist, nota bene, keines der Schuldenländer.

Als Regierungschef Papandreou am 31.10.2011 eine Volksabstimmung über das zweite Rettungspaket für Griechenland ankündigte, stoppten die anderen Euroländer die Auszahlung der nächsten Kredittranche und brachten Papandreou dazu, auf das Referendum zu verzichten und Platz für eine parteiübergreifende Übergangsregierung zu machen, die die Sparauflagen durchsetzen sollte. Schließlich wurde in Italien die Regierung Berlusconi kurz darauf von einem Expertenkabinett abgelöst.

Im Jahr 2012 mehren sich allerdings die Zeichen einer euroskeptischen Gegenbewegung von links und rechts gegen die technokratische Rettungspolitik. Im Februar wird die Verabschiedung des griechischen Sparprogramms von Generalstreik und schweren Krawallen begleitet. Im Mai verlieren die alten Volksparteien Pasok und Nea Demokratia, die das Sparprogramm tragen, bei den Parlamentswahlen massiv; den größten Zuwachs erlebt die linkspopulistische Partei „Syriza“ mit einem Wahlkampf gegen das Sparpaket. In den Niederlanden zerbricht im April die Regierung Rutte, weil ihr die rechtspopulistische „Partei für die Freiheit" wegen eines Sparprogramms die Unterstützung entzieht. In Frankreich erobern die Sozialisten das Präsidentenamt und die Nationalversammlung ebenfalls mit einem Programm, das gegen die strikte Sparpolitik gerichtet ist. In Deutschland nehmen die Klagen von links und rechts gegen die institutionellen Reformen vor dem Bundesverfassungsgericht zu. Die Krisenpolitik der EU ist damit nicht nur strittiger geworden, sondern erlebt eine Politisierung von unten. 
In der öffentlichen Meinung der Euroländer sind die Unterstützung für den Euro und das Vertrauen in die EU in der Eurokrise merklich geschrumpft. ${ }^{12}$ In einer im Mai 2012 veröffentlichten Umfrage findet das Pew Research Center in keinem der untersuchten Euroländer eine Mehrheit, die den Euro für „eine gute Sache " hält. ${ }^{13}$ Der in der Eurokrise zutage tretende Verteilungskonflikt zwischen Sparern und Rettern politisiert die Geldpolitik. In den Schuldenländern stehen die Regierungen unter dem Druck, harte Opfer von Gesellschaften zu verlangen, die ohnehin durch Rezession und hohe Arbeitslosigkeit geplagt sind. Regierungen in Deutschland und den anderen Länder der Sparer-Koalition sind in ihrem Beistand für die Schuldenländer auch durch die schwache transnationale Identität und Solidarität ihrer Wähler eingeschränkt.

$\mathrm{Ob}$ sich die vom neofunktionalistischen Spillover- und Pfadabhängigkeitsprozesse „von oben “ oder aber die postfunktionalen, euroskeptischen Politisierungstendenzen „von unten“ am Ende durchsetzen werden, ist eine offene Frage. Bisher jedenfalls scheint die neofunktionalistische Logik zu dominieren. Die gleichen Befragten, die mehrheitlich den Euro nicht für eine gute Sache halten, wollen andererseits mehrheitlich nicht zu ihren nationalen Währungen zurückkehren. ${ }^{14}$ Die irischen Wähler, die in der Vergangenheit wenige Chancen ausgelassen haben, um EU-Verträge abzulehnen, stimmten dem Fiskalpakt mit deutlicher Mehrheit zu. Die griechischen Wähler haben bei den Neuwahlen zum Parlament im Juni 2012 den Unterstützern des Sparabkommens mit der EU wieder eine Mehrheit verschafft. Die öffentliche Meinung scheint von der gleichen Pfadabhängigkeit geprägt zu sein wie die Regierungspräferenzen.

Die Differenzierung der Integration, also die von Politikbereich zu Politikbereich unterschiedliche Partizipation von Mitgliedstaaten, hat sich in der Eurokrise ebenfalls nicht verschärft. Die WWU war von Anfang an der am stärksten differenzierte Politikbereich der EU. Dass Großbritannien und Tschechien beim Fiskalpakt nicht dabei sind, setzt die euroskeptische Politik dieser Länder lediglich fort. Bemerkenswerter ist es, dass sich Dänemark und Schweden, wo der Euro an der euroskeptischen Bevölkerung gescheitert ist, zum Fiskalpakt bekennen.

\section{Schlussbemerkungen}

Drei Jahre nach ihrem Beginn erscheint die Eurokrise als Musterfall der supranationalistischen Integrationstheorie. Alle Zutaten der neofunktionalistischen Integrationsdynamik sind auszumachen: transnationale ökonomische Akteure als treibende Kräfte; funktionaler Spillover zwischen unterschiedlich stark integrierten, aber interdependenten Politikbereichen; supranationale Akteure, die ihre Tätigkeiten autonom erweitern; Pfadabhängigkeiten und endogene Präferenzen.

Für den Intergouvernementalismus, der die anfängliche Ausgestaltung der WWU plausibel erklären kann, spricht nach wie vor, dass die Regierungen - vor allem diejenigen Deutschlands und Frankreichs - die zentrale Rolle im Krisenma-

12 Eurobarometer 75 und 76, 2011.

13 Vgl. http://www.pewglobal.org/2012/05/29/european-unity-on-the-rocks/.

14 Vgl. Fussnote 11. 
nagement und Reformprozess spielen und dass ihre Positionen und Koalitionen sich gegenüber der Entstehungsphase der WWU nicht wesentlich verändert haben. Allerdings haben die Regierungen die Kontrolle über den Integrationsprozess verloren. Die Eurokrise resultiert aus den Konstruktionsmängeln, die die Regierungen der WWU in den 1990er-Jahren in die Wiege gelegt haben; sie wird durch transnationale Interdependenzen und Externalitäten zwischen Politikbereichen befeuert; und sie entwickelt Pfadabhängigkeiten, die die Staaten dazu zwingen, sich auf mehr Integration einzulassen, als sie ursprünglich wollten.

Aus konstruktivistischer Sicht zeigt die Krise, dass der Euro kein erfolgreiches Sozialisations- und Gemeinschaftsbildungsprojekt war. Weder hat sich die Fiskalpolitik der Euroländer merklich angenähert, noch haben sich durch den Euro transnationale Identität und Solidarität entwickelt. Diese ideellen Gemeinschaftsressourcen fehlen der Eurozone in der Krise; das Vertrauen in den Euro und in die EU als Ganzes sinkt; internationale Verteilungskonflikte rücken ins Bewusstsein; die euroskeptische Politisierung der Währungspolitik wächst. Allerdings hat die Schwäche gemeinsamer Ideen und europäischer Identität die Spillover- und Pfadabhängigkeitsmechanismen bisher nicht wirksam behindert.

Der Verlauf der Eurokrise legt nahe, dass sich die konkurrierenden Bedingungen und Mechanismen der Integration sequenziell entfalten: von der intergouvernementalistisch ausgehandelten anfänglichen Integration über die Spillover-Prozesse und Pfadabhängigkeiten zum populistischen Backlash (vgl. Leuffen et al. 2012). Anfangs dominierte in der Ausgestaltung der WWU die intergouvernementale Logik: im Konflikt und in den Verhandlungen zwischen den Regierungen der EU setzte sich der Staat mit der größten Verhandlungsmacht durch. Damit wurden erst die Bedingungen dafür geschaffen, dass die neofunktionalistische Logik sich entfalten konnte: vermehrte transnationale finanzielle Interdependenz, „sunk costs" der Währungsintegration, eine autonome supranationale Organisation wie die EZB, negative Externalitäten zwischen Geld- und Fiskalpolitik.

In der Krise transformierten diese Bedingungen die ursprüngliche Präferenzkonstellation und die Verhandlungsdynamik. Es bildete sich eine endogene Präferenz, den Euro zu verteidigen, und dieses gemeinsame Interesse genoss Priorität gegenüber den fortbestehenden nationalen Interessenunterschieden. Damit entstand im Kern ein „Feiglingsspiel“, in dem die Sparkoalition und die Beistandskoalition zwar versuchen, ihre unterschiedlichen Präferenzen für die Eurorettung durchzusetzen, aber vor dem Zerfall des Euro und der Eurozone zurückschrecken. Das Ergebnis ist ein nicht-beabsichtigter Integrationsfortschritt: die ursprünglich bewusst dezentralisierte Fiskalpolitik wurde durch gemeinsame Finanzierungsinstitutionen (EFSF und ESM) und durch eine verschärfte, supranationale Kontrolle der nationalen Haushaltspolitik stärker zentralisiert.

Diese Zentralisierung schuf in einem dritten Schritt die Voraussetzungen für die Entfaltung der postfunktionalen Logik. In der Eurokrise greift „Europa“ in einem ungekannten Ausmaß in die Autonomie der Mitgliedstaaten und den Alltag der Bürgerinnen und Bürger ein. Die Sparprogramme und Haftungsverpflichtungen schaffen hohe (aktuelle und potenzielle) Belastungen in den Gesellschaften sowohl der Schuldenländer als auch der Beistandsländer, und Verteilungskonflikte zwischen ihnen, die nicht durch transnationale Identitäten gedeckt sind und das 
Vertrauen in die supranationalen Institutionen angreifen. Das politisiert die Währungsunion und mobilisiert den euroskeptischen Populismus links wie rechts. Der weitere Verlauf der Eurokrise wird unter anderem davon abhängen, ob die postfunktionale Logik die neofunktionalistische Logik verdrängen wird und Differenzierung, Stagnation und Zerfall an die Stelle von Integrationsfortschritten treten werden.

\section{Literatur}

Belke, Ansgar. 2011. Als letzter Ausweg droht Inflation. http://www.faz.net/aktuell/ wirtschaft/europas-schuldenkrise/die-gefahr-einer-neuen-krise-als-letzter-ausweg-drohtinflation-1577953.html.

Bieling, Hans-Jürgen, und Marika Lerch. (Hrsg.) 2006. Theorien der europäischen Integration. Wiesbaden: VS Verlag für Sozialwissenschaften.

Dyson, Kenneth, und Kevin Featherstone. 1999. The Road to Maastricht. Negotiating Economic and Monetary Union. Oxford: Oxford University Press.

Enderlein, Henrik. 2012. Denn sie wissen, was sie tun. http://www.handelsblatt.com/meinung/gastbeitraege/gastkommentar-denn-sie-wissen-was-sie-tun-seite-all/6189038-all. html.

Grieco, Joseph M. 1996. State Interests and International Rule Trajectories: A Neorealist Interpretation of the Maastricht Treaty and European Economic and Monetary Union. Security Studies 5:261-306.

Haas, Ernst B. 1968. The Uniting of Europe: Political, Social and Economic Forces, 195057. Stanford: Stanford University Press.

Hix, Simon. 1994. The Study of the European Community: The Challenge to Comparative Politics. West European Politics 17:1-30.

Hooghe, Liesbet, und Gary Marks. 2008. A Postfunctionalist Theory of European Integration: From Permissive Consensus to Constraining Dissensus. British Journal of Political Science 39:1-23.

Jachtenfuchs, Markus, und Beate Kohler-Koch. 1996. Regieren im dynamischen Mehrebenensystem. In Europäische Integration. Hrsg. Markus Jachtenfuchs und Beate KohlerKoch, 15-44. Opladen: Leske + Budrich.

Keohane, Robert O. 1984. After Hegemony: Cooperation and Discord in the World Political Economy. Princeton: Princeton University Press.

Leuffen, Dirk, Berthold Rittberger und Frank Schimmelfennig. 2012. Differentiated Integration. Explaining Variation in the European Union. Basingstoke: Palgrave.

Lewis, Jeffrey. 2010. How Institutional Environments Facilitate Co-operative Negotiation Styles in EU Decision-making. Journal of European Public Policy 17:648-664.

Marks, Gary, Liesbet Hooghe und Kermit Blank. 1996. European Integration since the 1980s: State-Centric versus Multi-Level Governance. Journal of Common Market Studies 34:343-378.

Moravcsik, Andrew. 1993. Preferences and Power in the European Community: A Liberal Intergovernmentalist Approach. Journal of Common Market Studies 31:473-524.

Moravcsik, Andrew. 1998. The Choice for Europe. Social Purpose and State Power from Messina to Maastricht. Ithaca: Cornell University Press. 
Moravcsik, Andrew. 2005. The European Constitutional Compromise and the Neo-functionalist Legacy. Journal of European Public Policy 12:349-386.

Parsons, Craig. 2003. A Certain Idea of Europe. Ithaca: Cornell University Press.

Pierson, Paul. 1998. The Path to European Integration: A Historical-Institutionalist Analysis. In European Integration and Supranational Governance. Hrsg. Wayne Sandholtz und Alec Stone Sweet, 27-58. Oxford: Oxford University Press.

Schimmelfennig, Frank. 2010. The Normative Origins of Democracy in the European Union: Toward a Transformationalist Theory of Democratization. European Political Science Review 2:211-233.

Schimmelfennig, Frank. 2012. Constructivist Perspectives. In The Oxford Handbook of the European Union. Hrsg. Erik Jones, Anand Menon und Stephen Weatherill, 34-47. Oxford: Oxford University Press.

Schmitter, Philippe C. 1969. Three Neo-Functional Hypotheses about International Integration. International Organization 23:161-166.

Stone Sweet, Alec und Wayne Sandholtz. 1997. European Integration and Supranational Governance. Journal of European Public Policy 4:297-317.

Straubhaar, Thomas 2011. Drei Euro-Zukunftsszenarien. Die Volkswirtschaft 11:30-33.

Wolf, Dieter, und Bernhard Zangl. 1996. The European Economic and Monetary Union: "Two-Level Games" and the Formation of International Institutions. European Journal of International Relations 2:355-393.

\section{Autorenangaben:}

Prof. Dr. Frank Schimmelfennig, ETH Zürich, Center for Comparative and International Studies, IFW D 45.1, Haldeneggsteig 4, 8092 Zürich, Schweiz, frank.schimmelfennig@eup.gess.ethz.ch 